\title{
SEMA4A: An ontology for emergency notification systems accessibility
}

\author{
A. Malizia*, T. Onorati, P. Diaz, I. Aedo, F. Astorga-Paliza \\ Universidad Carlos III de Madrid, Dpto. Informatica, Avda. de la Universidad, 30, 28911 Leganés, Madrid, Spain
}

Keywords:

$\mathrm{HCI}$

Accessibility

Ontology management

Universal design

Emergency response information systems (ERIS)

\begin{abstract}
A B S T R A C T
Providing alert communication in emergency situations is vital to reduce the number of victims. Reaching this goal is challenging due to users' diversity: people with disabilities, elderly and children, and other vulnerable groups. Notifications are critical when an emergency scenario is going to happen (e.g. a typhoon approaching) so the ability to transmit notifications to different kind of users is a crucial feature for such systems. In this work an ontology was developed by investigating different sources: accessibility guidelines, emergency response systems, communication devices and technologies, taking into account the different abilities of people to react to different alarms (e.g. mobile phone vibration as an alarm for deafblind people). We think that the proposed ontology addresses the information needs for sharing and integrating emergency notification messages over distinct emergency response information systems providing accessibility under different conditions and for different kind of users.
\end{abstract}

\section{Introduction}

Within an emergency scenario sharing information and com mon knowledge about types of disasters, kinds of affected entities (people, infrastructures, communications, ...), measures and alerts (depending on the kind of emergency) is crucial in order to reduce the number of victims or damages.

Information technology (IT) is a relevant support when an emergency occurs, or is going to occur; furthermore IT tools like emergency response information systems (ERIS) can manage com munications and information processing, can help in decision mak ing and improve situational awareness when used in an emergency scenario. Technologies involved in ERIS can vary from mobile de vices, to client server applications, reaching complex distributed services architectures (Van de Walle \& Turoff, 2007). Nevertheless, one of the most relevant features of ERIS is alert notification since it can affect the final users and their safety.

Our approach consists of automatically adapting (with the use of our ontology) the notification of alerts to different kind of users (elderly, disabled, ...) depending on the type of technologies (de vices) they can access and considering the impact of the disaster on alerts communication and infrastructures. So for instance, when a fire is occurring in a building we know that we should communi cate with people by audio notifications instead of visual ones since smoke can reduce visibility, but also that we could use the same

* Corresponding author. Address: Alessio Malizia, Universidad Carlos III, Dpto Informatica, Avda de la Universidad 30, 28911 Leganes, Spain.

E-mail addresses: alessio.malizia@uc3m.es (A. Malizia), tonorati@inf.uc3m.es (T. Onorati), pdp@inf.uc3m.es (P. Diaz), aedo@ia.uc3m.es (I. Aedo). kind of alert for compensating disabilities, e.g. alerting blind users of a critical or dangerous event.

To provide emergency notifications is important but not trivial since in order to get an efficient communication many systems should interoperate with each other sharing a common knowledge with different terminologies and types of crisis or emergencies.

From the semantics point of view, a common language (at least a glossary) is needed in order to have coordination among users, systems and communications. Thus, it is important to codify semantics in an accessible way so that it is easy for users to inter pret notifications and for expert users to communicate among each other on relevant topics.

To help in augmenting the interoperability among systems, and also among people, involved in such scenarios we developed an ontology $^{1}$ called SEMA4A (Simple Emergency Alerts 4 [for] All) including concepts taken from emergency systems and control rooms but also from accessibility guidelines and interactive devices. The scope of this ontology is designing a common knowledge within ERIS and accessibility. Tim Bernes Lee stated (Berners Lee, 2007): "Disaster response is much about preparedness. If much relevant data is available in RDF, when a disaster strikes, those on the ground and across the world will be able to use it to know what best to do to respond". That's why we not only designed and developed our ontol ogy integrating the categories of information described above, but also used the Web Ontology Language (OWL) to adhere to a standard codification and to offer an interoperable knowledge framework for enabling collaboration among different ERIS. The novelty of this

\footnotetext{
${ }^{1}$ An ontology is defined (Neches et al., 1991) as a set of basic terms and relationships of a vocabulary within an area or subject, as well as the rules to combine terms and relations that extend the vocabulary.
} 
work is both in providing accessible emergency notifications and supporting systems interoperability at syntactic and semantics lev els by sustaining standard ontology codification and concepts map ping among different domains of accessibility and emergencies.

In Section 2 we will describe related works on $\mathrm{HCI}$, accessibility and emergency response information systems. Successively, in Sec tion 3, we will present the proposed approach describing our ontology driven methodology for semantic adaptation of emer gency notifications to different types of users. Section 4 will be about evaluating the proposed ontology, while in Section 5 we comment on obtained results and describe future works.

\section{Background}

\subsection{HCI and emergency}

Managing risk is crucial for Governments as well as for engag ing stakeholders, and providing accurate information to allow pub lic departments to make decisions on how to deal with risk (UK Resilience $^{2}$ ). Providing effective communication of alerts and emer gency situations is really important in order to reduce the number of victims. Reaching this goal is challenging because there is no effec tive standard for design due to different people characteristics: we considered users having different abilities, backgrounds, experi ences, ages and sizes. During an emergency situation, all people have in some sense a kind of disability, some of them might be caused by stress, the environment or even by lack of information.

Thus, it is important to design systems that effectively provide information to people included in vulnerable groups. This can be achieved by adopting Universal Design principles (Dix, Finley, Abowd, \& Beale, 2003).

As said by Information \& Communications Technologies Standards Board, there are two ways to reduce the gap between products and human abilities: the Design for all approach and Assistive Technol ogy (ICTSB, 2000). Aspects related to the implementation of Design for All in ICT from a developer oriented perspective have been also presented in Burzagli, Emiliani, and Gabbanini (2009).

\subsection{Accessibility standards}

The problem of the accessibility is only partially related to adopting measures that compensate the disadvantages or that sur pass the functional limitations of people with disabilities. In fact, adapting the environment to be accessible, not only is good for people with disabilities but also for those without disabilities, this is remarkable if we think about urban adaptation in big cities.

One of the most important communication channels is the Internet and thus it is important to provide accessibility over the web. In order to ensure web accessibility, web developers and designers can follow guidelines established by the World Wide Web Consortium (W3C), through a special working group called Web Accessibility Initiative; these guidelines are called Web Con tent Accessibility Guidelines (WCAG) (WAI, 2006).

It is also important to examine the approach taken by US, the so called Section $508,{ }^{3}$ which aims at adapting information to people with disabilities.

To verify the compliance of a specific web content to guidelines, developers may use accessibility checker software, such as: Bobby, LiFT, A Prompt (A Prompt, 2006; Bobby, 2006; LiFT, 2006); this is not sufficient since these tools mainly perform a syntactic assess ment of web pages, but they are not able to verify semantic con tents and to adapt them for special needs users (Yang et al., 2007).

\footnotetext{
2 http://www.ukresilience.info/.

3 http://www.section508.gov/.
}

We should mention that another interesting approach has been proposed by Gabrielli, Mirabella, Kimani, and Catarci (2004), Gabrielli, Mirabella, Teso, and Catarci (2005), where authors provided an accessibility approach to e learning for people with special needs. In their work, they highlighted a set of abilities together with a set of visual features by which learners, with differ ent abilities, could interact with an e learning system. We studied these difficulties since they are similar to the ones we found when adapting (or designing how to adapt) information to different kinds of users.

\subsection{Emergency systems}

In this section, we analyse information systems used to inform people about emergencies. These systems are called Emergency Notification Systems (ENS) and are included within Emergency Re sponse Information Systems (ERIS).

In these days, many emergency notification systems exist and are used to notify people in places like home, office, school, out door, etc. Notifications are often delivered by phone, e mail or websites. Some systems also permit to deliver messages to pagers, faxes, VoIP (Voice over IP), SMSes (Short Message Service), and in stant messengers; nevertheless, these are features that today are already included in mobile phones, smart phones or PDA (Personal Digital Assistant).

In order to understand if existing ENS consider Accessibility principles, we studied the systems included in Table 1 .

After surveying the systems presented in Table 1, we found out that ENS are used by private companies, schools, government offi ces, Red Cross, fire fighters, police, as well as many other institu tions. Service technologies provided by these systems are almost the same (aee 'Notification' column).

We found out that Waves Alerter was the only system providing accessible notifications; in particular for people with auditory defi ciencies using TDD/TTY, ${ }^{4}$ but this technology is old and non stan dard. Therefore, even if ENS are intended to inform people about an emergency, we found out that these systems do not provide noti fications in a format considering people's profiles and preferences. This can be done by providing ENS with a model or base of knowl edge (an ontology in our case) that reflects this information (users' profile and preferences), as well as information related to accessibil ity, media and emergencies in order to provide effective and custom ized emergency notifications.

Moreover, we have also taken into account the CAP (Common Alerting Protocol) 1.0 specification approved by the OASIS consor tium. OASIS ${ }^{5}$ is the Organization for the Advancement of Structured Information Standards, and CAP is an XML based data format for interchanging warnings and emergencies between alerting technol ogies. The scope of the CAP is focused on defining and exchanging the different kinds of alerts and types of notifications but does not take into account the different abilities of the users and does not, explicitly, model the relationships among the technologies and the kinds of emergencies. So we decided to use, directly, a structured knowledge in form of ontology to link all the dimensions of the alert's notifications information space (types of notifications, users' abilities to react or understand the notifications, available technolo gies, types of emergencies and impact of the emergency on the avail able technologies). This is exactly about supporting software interoperability since CAP is a standard way of communicating about emergency notifications within systems. We highlight that by inte grating the CAP data structures into our ontology we also obtained

\footnotetext{
${ }^{4}$ (Telecommunications Device for the Deaf/TeleTYpewriter) A user terminal with keyboard input and printer or display output used by the hearing and speech impaired (source: PCMag.com, http://www.pcmag.com/).

${ }^{5}$ http://www.oasis-open.org/home/index.php.
} 
Table 1

Comparative survey of accessibility features in emergency notification systems.

\begin{tabular}{|c|c|c|c|c|c|}
\hline System & Web & Comm. Type & Source & Notification & Accessibility \\
\hline $3 n$ & http://www.3nonline.com/ & $\begin{array}{l}\text { Emergency, situational } \\
\text { alarm, alert, system status }\end{array}$ & Phone, Web & $\begin{array}{l}\text { Phone, E-mail, Pager, Fax, SMS, } \\
\text { PDA }\end{array}$ & No \\
\hline AlertFind & $\begin{array}{l}\text { http://www.messageone.com/ } \\
\text { crisis-communications/ }\end{array}$ & $\begin{array}{l}\text { Emergency, situational } \\
\text { alarm, alert }\end{array}$ & Phone, Web & $\begin{array}{l}\text { Phone, E-mail, Pager, Fax, SMS, } \\
\text { PDA }\end{array}$ & No \\
\hline Arce & $\begin{array}{l}\text { https://arce.dei.inf.uc3m.es/ } \\
\text { arce_demo/ }\end{array}$ & $\begin{array}{l}\text { Emergency, situational } \\
\text { alarm, alert, system status }\end{array}$ & E-mail, Web & Web pages, E-mail & No \\
\hline $\begin{array}{l}\text { Command } \\
\text { Caller }\end{array}$ & $\begin{array}{l}\text { http://www.voicetech.com/ } \\
\text { Command_Caller_40.htm }\end{array}$ & $\begin{array}{l}\text { Emergency, situational } \\
\text { alarm, alert }\end{array}$ & Phone, E-mail, Fax & $\begin{array}{l}\text { Phone, E-mail, Pager, Fax, SMS, } \\
\text { PDA }\end{array}$ & No \\
\hline RapidReach & http://www.rapidreach.com/ & $\begin{array}{l}\text { Emergency, situational } \\
\text { alarm, alert }\end{array}$ & Phone, Web & Phone, Pager, Fax, SMS y E-mail & No \\
\hline Sahana & http://www.sahana.lk/ & Emergency & Web & Web pages & No \\
\hline Sigame & http://www.sigame.es/ & Emergency & Web & Web pages & No \\
\hline SWN & $\begin{array}{l}\text { http://www.sendwordnow.com/ } \\
\text { smart_alert_service.aspx }\end{array}$ & $\begin{array}{l}\text { Emergency, situational } \\
\text { alarm, alert, system status }\end{array}$ & $\begin{array}{l}\text { Phone, E-mail, SMS, } \\
\text { Blackberry, Palm, Web }\end{array}$ & $\begin{array}{l}\text { Phone, E-mail, Pagers, SMS, MMS, } \\
\text { VoIP, Skype, Chat y PDAs }\end{array}$ & No \\
\hline $\begin{array}{l}\text { WAVES } \\
\text { Alerter }\end{array}$ & $\begin{array}{l}\text { http://www.madah.com/products/ } \\
\text { subpage.asp?mer_notf_sys }\end{array}$ & $\begin{array}{l}\text { Emergency, situational } \\
\text { alarm, alert, system status }\end{array}$ & Phone, Web & $\begin{array}{l}\text { Phone, E-mail, Fax, PDA and TDD / } \\
\text { TTY }\end{array}$ & Yes \\
\hline
\end{tabular}

interoperability at semantics level because we linked concepts al ready present in our ontology to the hierarchical structure extracted from the CAP.

\section{Approach}

In order to deploy an accessible emergency notification system, our proposal focuses on developing an ontology that adapts notifi cations by using accessibility and usability concepts.

This can be done by means of a knowledge base that reflects users' needs as well as ways for effectively present them informa tion according to their needs, the kind of emergency, and available technologies (depending on the users' abilities and the type of emergency). Having such information base, assures us to be aware of stakeholders' characteristics and needs; making it possible to reach more people.

Designing such a complex system requires an articulated knowledge base that consists of knowledge in three areas: accessi bility, user profiles, and devices. For these reasons we chose to model the knowledge base by an ontology which can include com plex relationships among concepts and can provide tools like first order logic to verify the validity and integrity of the knowledge codified within it.

Ontologies provide a semantic resource to describe information related to a specific domain. Ontologies will reduce the necessary effort to specify accessibility requirements for emergency notifica tion systems because there will be a set of shared concepts that could be used for specifying requirements.

An ontology driven system could be developed on top of this codified knowledge that could be used to infer and adapt notifica tions in emergency scenarios depending on users' special needs (e.g. a fire alarm in a building could cause the system to send a short video to deaf people showing the emergency exit locations to solve the problem of not hearing the alert sound).

For these reasons, we studied different existing ontologies for accessibility and used information from these ontologies to render such concepts for adapting emergency notifications to different types of users.

\subsection{Ontology and accessibility}

To clarify the way in which ontologies can be used in the field of accessibility, in this section we describe three works that are rele vant to us and related to our approach.

The first is the $\mathrm{KAICO}^{6}$ system, which uses the OntoSaw ontology, the second is the Dante tool that uses the Web Authoring for Acces

\footnotetext{
${ }^{6}$ http://quercusseg.unex.es.
}

sibility (WAfA) ontology, and finally, we analise the AccessOnto ${ }^{7}$ architecture and ontology.

OntoQuercus group, sub group of Quercus Software Engineer ing Group (Quercus, 2007) of University of Extremadura developed the KAICO system, which is composed by an ontology as knowl edge base, software applications that serve to add semantic tags to web pages elements, applications to extract information and specialised hardware to communicate with blind people (Lozano Tello, Macías, Prieto, Sánchez, \& Sosa, 2004; Lozano Tello, Macías, Sánchez, \& Sosa, 2003).

The OntoSaw ontology contains the conceptual model of ele ments that form web pages; the ontology is oriented to represent the necessary information so that the pages are accessible. With the purpose of identifying elements and attributes of the ontology, opinions from people with visual disability were collected, to gether with observation of difficulties faced by them while visiting web sites with poor accessibility. In order to complement this ontology, elements and characteristics identified in WCAG 1.0 have been taken into account.

This ontology has been constructed following the METHONTOL OGY methodology (Pinto \& Martins, 2002), using Protégé 2000, ${ }^{8}$ and represented by the DARPA Agent Markup Language and Ontol ogy Inference Layer or Ontology Interchange Language, the DAM $\mathrm{L}+\mathrm{OIL}^{9}$ language.

Another relevant work comes from the Information Management Group of computer science department at University of Manches ter, that has designed Dante ${ }^{10}$ (Yesilada, Harper, \& Goble, 2004), which is a semiautomatic tool that aims at improving navigation be tween web pages for people with visual deficiencies. The main objec tives of Dante are: to analyze web pages in order to identify objects which improve the navigation, to translate into page elements the concepts from WAfA ontology, and to transform (transcode) ${ }^{11}$ the pages using these annotations so that they can be easily accessed using a screen reader.

The WAfA ontology (Web Authoring for Accessibility ${ }^{12}$ ), is also known as the Travel Ontology because it is based on the analogy of tourists' trips with web navigation; it represents concepts and rela tions necessary to automatically model the structural organization and navigation of web pages (Yesilada Y., 2005). In Dante, this ontol ogy is implemented as a controlled vocabulary describing annota tions and transformations.

\footnotetext{
${ }^{7}$ http://shapevle.cant.ac.uk/AccessOntoTool.htm.

8 Ontology Editor. http://protege.stanford.edu/.

${ }^{9}$ http://www.daml.org/language/.

10 http://dante.cs.manchester.ac.uk/.

11 www.w3.org/1999/07/NOTE-annot-19990710.

12 http://augmented.man.ac.uk/ontologies/wafa.owl.
} 
A different approach has been taken at the Businesses school at the Canterbury Christ Church University. AccessOnto has been devel oped (Masuwa Morgan \& Burrell, 2004) as a tool for requirements engineering managed as a repository of semantic requirements for accessibility (Guarino, 1997). The goal of this ontology is to extract a specification of requirements by using a knowledge base built on user's characteristics. The repositories used by this system in cluded two kinds of knowledge: declarative knowledge focused on a limited set of classes defined by the guidelines, interface ob jects and user's characteristics, and procedural knowledge com posed of production rules capturing the ideas of adaptive programming and multiple relations management (entities and dependencies).

The guidelines included in AccessOnto are proposed by: WCAG, Sun Micro System, IBM, Microsoft and Apple. Neverthe less, the intention is that in the future the repository will point directly to the sources of guidelines. At the moment, the prob lem is that existing guidelines do not use a standard format, which makes it difficult to integrate guidelines from different sources.

Our purpose was to explore if ontology can be used to specify accessibility requirements so that it was feasible to create an emergency notification system that used this knowledge to de cide the optimal media and format to adopt for notification messages.

The three described approaches are a useful foundation for our work but there are several constraints:

1. The Kaiko idea is to add semantic marks to Web pages elements in order to be able to present them to blind people. It is based on OntoSaw ontology that contains elements to model Web pages, attributes and their relations. Some limitations of this approach are:

- Generated code can present problems in being interpreted by some screen readers and browsers.

- The ontology is focused only on blind people.

2. Dante aims at improving the navigation among web pages for people with visual deficiencies. Dante is based on the WAfA ontology, which contains concepts and relationships needed to model organization, structures and navigation of web sites. WAfA defines concepts about the rendering of objects in a web page (structural properties) and how these objects are used, in other words, WAfA encapsulates exten sive knowledge to make explicit structural and navigation information of a web page. This approach is more complex than the one made by Kaiko, nevertheless it presents some problems:

- Existing pages are transformed into small fragments that could cause the loss of users' context.

- Screen readers could have difficulties reading the web pages annotations. For this reason it might occur that screen read ers present wrong information to users or cannot be able to interpret the entire code.

- The ontology is only focused on people with visual disabilities.

3. AccessOnto is a requirements engineering tool in form of an accessibility requirements repository. This ontology is made of three information repositories: user profile repository, guide lines repository and interface object action repository. Access Onto also presents some weak points:

- Structural information given by previous ontologies is not included.

- The ontology is at an early stage, reason why it is not formal ized using a standard language like OWL.

After reviewing the existing approaches, we can point out that ontologies will clearly help the development of emergency notifi cation systems by fulfilling accessibility and universal design prin ciples; nevertheless there is a strong need of integrating or mapping information among them in order to take advantage of the various ontologies characteristics.

The current version of SEMA4A includes information related to content design, accessibility guidelines, emergencies, devices and communication technologies organized in three main categories: (1) WAfA; (2) AccessOnto; (3) EMEDIA. Below we explain what information is included in each section, as well as the process fol lowed in order to model and integrate this information.

\subsection{The proposed ontology}

Designing and developing ontologies is a complex task involv ing knowledge management and domain experts (Pinto \& Martins, 2002). Ontologies have proliferated in these last years mainly thanks to the semantic web development. They have been widely adopt in other areas like e learning for adaptation purposes (Cristea, 2004; Pattuelli, 2008). Having this in mind, we developed the SEMA4A ontology, which has been created using the Web Ontology Language (OWL) as well as an OWL reasoning tool from Mindswap laboratory at Maryland University called Pellet to verify the consistency of existing classes in the ontology.

Our purpose was to model a set of sharable concepts and knowledge considering a successive step the rigorous formaliza tion and the development of an expert system based on the pro posed ontology as suggested by Geller, Perl, and Lee (2004).

The first part of the proposed ontology is EMEDIA (Emergency and MEDIA technologies); which is the portion of the SEMA4A ontology that provides concepts and relations about emergency and media technologies. We developed it through a semiautomatic procedure with two phases: the first phase was performed to ex tract concepts and relations concerning emergency and media technologies; the second one consisted of integrating new infor mation within the existing ontology (adding relations with the others portions). We applied this technique to develop and expand the parts of our ontology related to the emergencies; we have also modelled how technologies affect accessibility.

The first phase of our procedure consisted of, automatically, extracting concepts and relations from WordNet (Miller, Beckwith, Felbaum, Gross, \& Miller, 1990). The starting point was a simple set of words, related to emergency and media technologies, found in MyFlorida.com (The Official Portal of the State of Florida. MyFlori da.com Taxonomy Disasters \& Emergency Information ${ }^{13}$ ) and A Simple Taxonomy for Mobile Emergency Announcement Systems (Addams Moring et al., 2005), as suggested by emergency field experts (Spanish civil protection). We proceeded, initially, retrieving these terms in WordNet. For each meaning of each term, WordNet gave a set of synonyms, holonyms, hypernyms, hyponyms and meronyms. Synonyms represented new concepts that could be possibly added to the ontology. Examples of concepts used as a seed for retrieving re lated knowledge are: Avalanche, Blizzard, Colds, Cyclone, Drought, Earthquake, Epidemic, Eruption, Fire, Flood, Forest fire, Hailstorm, Heat wave, Hurricane, Ice storm, Lahars, Landslides, Limnic eruption, Maelstrom, Mudslide, Seiche, Sinkholes, Storm, Thunderstorm, Tor nado, Tsunami, Typhoon, Volcano, and Wildfire.

We iterated this procedure with all synonyms found as in an $\mathrm{n}$ ary tree: each concept was a node having a child for each related synonym until a maximum of three levels (this threshold has been experimentally set; fewer levels generated few terms, while more levels added terms which were not really related to our domain). For each concept we stored all meanings and

13 http://www.myflorida.com/taxonomy/floridian/disasters\%20\&\%20emergency\%20information/. 
relations. In this way, we obtained a new taxonomy related to emergency and media. The final phase consisted of integrating the new taxonomy in the existent ontology as a new class, EMEDIA.

After creating EMEDIA we extracted, refined, upgraded and linked information obtained from: (1) an ontology that contains concepts and relations needed to model the organization, structure and navigation of information contents (WAfA); (2) an ontology that includes accessibility guidelines, user's profiles and actions that can be performed by users with different abilities (AccessOnto).

In particular the basic classes included in this combination of ontologies are: Impairment, Age, and Expertise. Impairments class includes: Motor (coordination difficulty, reach limitations, no tac tile sensation), Visual (color blindness dichromatic and color tones, low vision, blindness, deafblind), Cognitive (word and spatial dys lexia, learning difficulty), Hearing (deafness, deafblind). Age is sub divided in children and elderly (with their combination of disabilities and characteristics). Expertise is subdivided into: nov ice, intermediate, and expert; it includes all kind of disabilities originated by the level of computer education users have.

We combined these two existing ontologies by including and mapping their concepts and relations into our ontology together with the EMEDIA part. We used different transformations and semantics paths to link related concepts in these that before were separated ontologies, thus creating a common knowledge base on accessibility guidelines and users' characteristics and abilities.

Summarizing, SEMA4A counts on three basic classes: WAfA, AccessOnto and EMEDIA; it includes information related to con cepts and relations needed to model organization, structure and navigation of information contents; it also includes accessibility guidelines, user's profiles and actions that users can perform, as well as, information related to emergencies, notifications and devices. These main classes are linked with relations exist ing within their subclasses. In the next section we provide a use case that depicts the more common relations that exist in our ontology.

Fig. 1 shows the high level classes that form the SEMA4A ontology.

\subsection{Use case}

In order to show how the concepts and relationships included in SEMA4A could be used by ENS for providing accessible alerts noti fications, we provide a use case.

Imagine a deafblind person walking alone on the streets in a city that he/she is visiting for the first time. Before arriving the city, this deafblind has been subscribed to an ENS.

Weather forecast for the city, where the deafblind person is touring, expects that a tornado arrives in around $6 \mathrm{~h}$. If the ENS wants to alert the person about this event, it is important to pro vide significant information in a format that he/she can access. When this person subscribed to the service, he/she communicated that the media device he/she carried was a Personal Digital Assis tant (PDA) with Internet connection. This person also pointed out that he/she had a special program installed in his/her device for reading the screen (screen reader) that transforms text and images into Braille writing system. The ENS could use SEMA4A for alerting the deafblind person in an accessible way of the upcoming event taking into account his/her preferences and profile information as follows:

- From the ontology, we have that deafblind is a visual and hear ing impairment where people cannot hear or see either partially or totally; we also have that people with this disability may have difficulties using a standard mouse (or a pointing device like the pen used with PDAs). It is common that deafblind people use speech input and speech output, sometimes with difficulties; they can also use tactile input and output (e.g. Braille line, which is a Braille display). It is also obtained from our ontology that deafblind people can use keyboards, and can notice vibrations. According to the actual version of SEMA4A, deafblind people can access text data using a Braille line (see Fig. 2).

- As the ENS needs to alert a deafblind person using a PDA with Internet access, about an approaching tornado, it obtains from our ontology that PDA (Personal Digital Assistant) is a media device that can communicate information contained in figures, sounds, text, as well as vibration signals (see Fig. 3 ). From our ontology it is also obtained that tornado is an emergency that can be communicated using the Internet, TV and radio (see Fig. 4). Moreover, SEMA4A defines that when a tornado alert should be communicated, this must be done following the Com mon Alerting Protocol (CAP) (see Fig. 5).

- According to the user's profile and preferences, it is desired that the ENS notifies via the Internet. Having this in mind, from the ontology we know that using the Internet we can communicate employing multiple languages, text, figure, video, sound or e mails, as shown in Fig. 6.

- In order to assure that this person can access the information, SEMA4A infers ontology fragments, as shown in Fig. 7, to follow some guidelines from: Web Accessibility Initiative (WAI), Acces sibility Quick Reference Guide, Custom Guidelines, Neuman's guidelines, as well as guidelines from IBM relative to adapt con tent for blindness.

- Finally, SEMA4A infers Web accessibility guidelines specific for deafblind, as well as specific Web elements (e.g. figures or images, sounds, input controls) that need to be formatted or transformed for assuring that this deafblind person can access the tornado alert notification over his/her PDA in order to save his/her life (Fig. 8). For instance images descriptions (which can be read by a text reader software) replacing graphics.

\section{Ontology evaluation}

\subsection{Evaluation criteria}

There exist many different methods and techniques to validate and evaluate ontologies as already described in the introduction. We used an approach inspired by Spyns, Meersman, and Jarrar (2002) based on triples extracted from the ontology which were defined as lexons. Formally, a lexon is as 5 tuple: $\langle(\mathrm{G}, \mathrm{L})$ : term 1 role co role term 2$\rangle$. Where $\mathrm{G}$ is the context and $\mathrm{L}$ is the language. Co role is the inverse of role; we can omit the $(G, L)$ pair and de scribe a lexon as a 4 tuple like $\langle$ President, directs, is directed by, enterprise $\rangle$. Usually only the role is explicitly represented (while the inverse is implicit), thus a lexon is described a triple, and in fact could be described as a combination of an OWL triple and its in verse, or as a conceptual graph style relation (Sowa, 1984).

Informally we can say that a lexon expresses that the term 1 (or head term) can have term 2 (or tail term) occur in an associating role with it. Conceptualisations can be represented in terms of lex ons. We can represent our ontology in form of a list of lexons.

The main research hypothesis in this evaluation is that lexons, representing the basic binary facts (contained in a corpus of docu ments or in ontology) expressed in natural language, can be ex tracted from the available textual sources, i.e. a corpus, using a specific parser, as well as, from our ontology as OWL triples. In fact in a specific domain noun phrases (NP) carry important informa tion about the domain itself, while verbs impose restriction on the nouns semantics. 


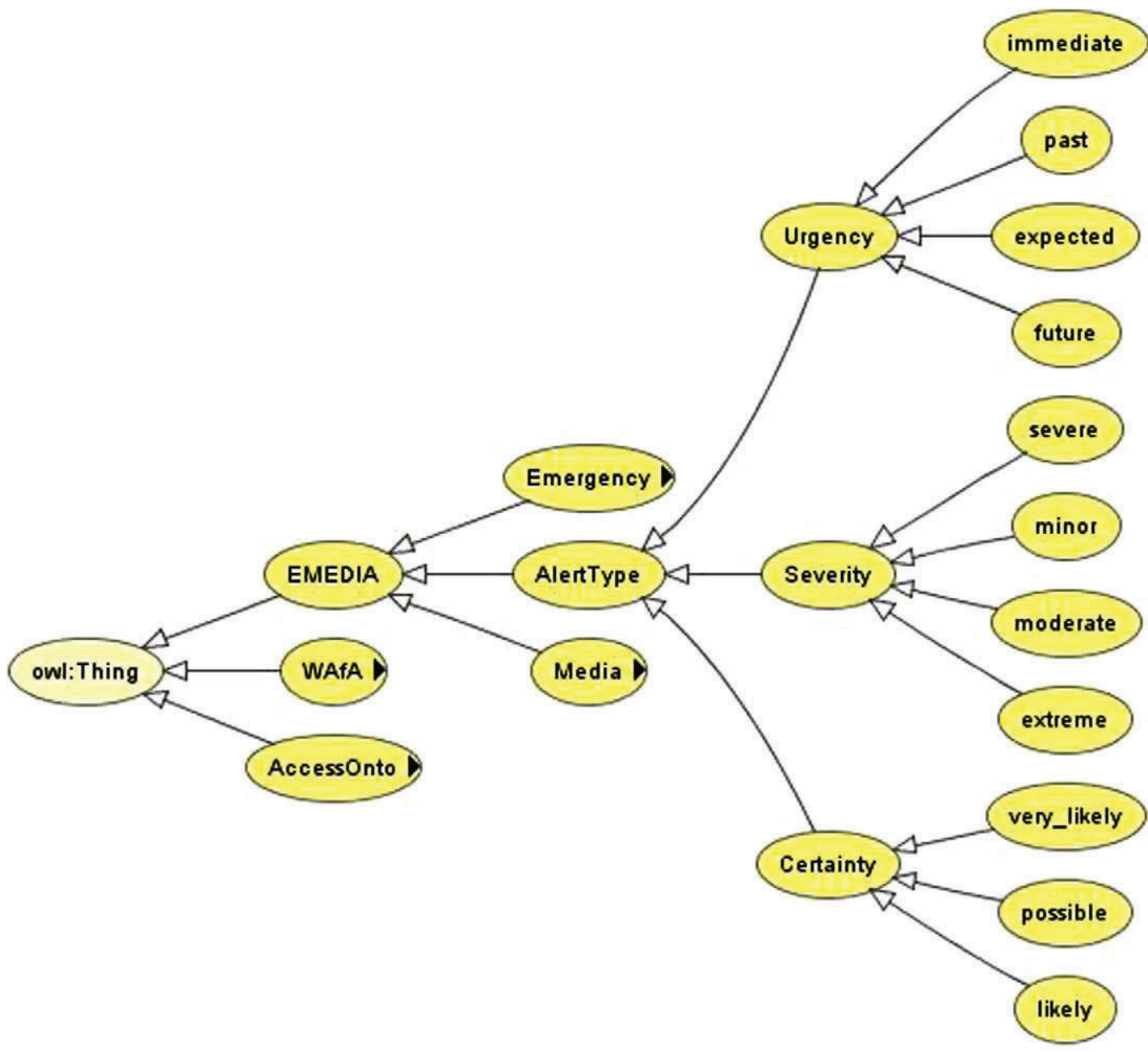

Fig. 1. SEMA4A ontology fragment.

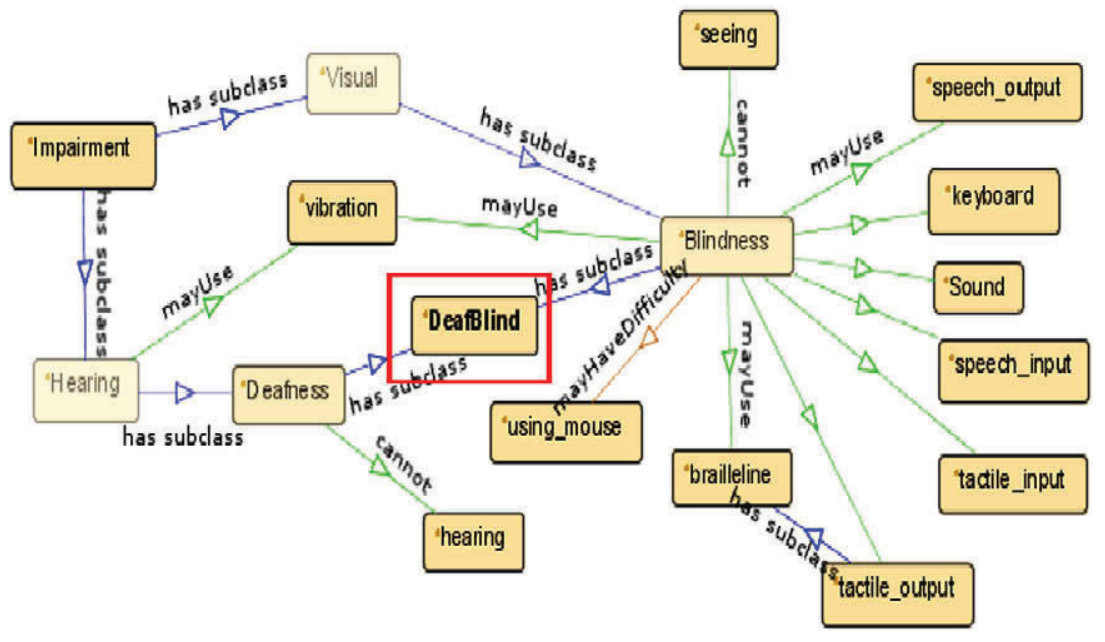

Fig. 2. Definition of deafblind.

The considered corpus is composed by articles about emer gency, accessibility and devices as suggested by the domains ex perts. In particular, for the emergency topic there are about 67 article from the proceedings of the conference ISCRAM2007 (Intelli gent Systems for crisis management), plus a manual developed by the North Central Texas regional government (Know what to do.
Think. Prepare. Act. ${ }^{14}$ ) and papers on community emergency man agement (for example: Schafer, Ganoe, \& Carroll, 2007). The total number of analysed words was about 300,000 for 500 pages. For

$\overline{14}$ http://www.knowwhat2do.com/en/pdf/KnoWhat2Do_Guide.pdf. 


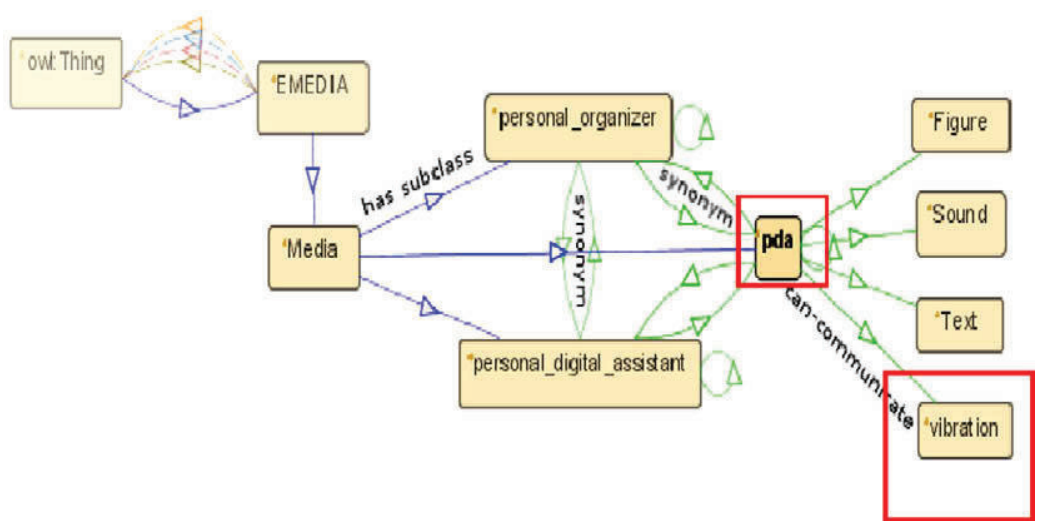

Fig. 3. PDA communication features.

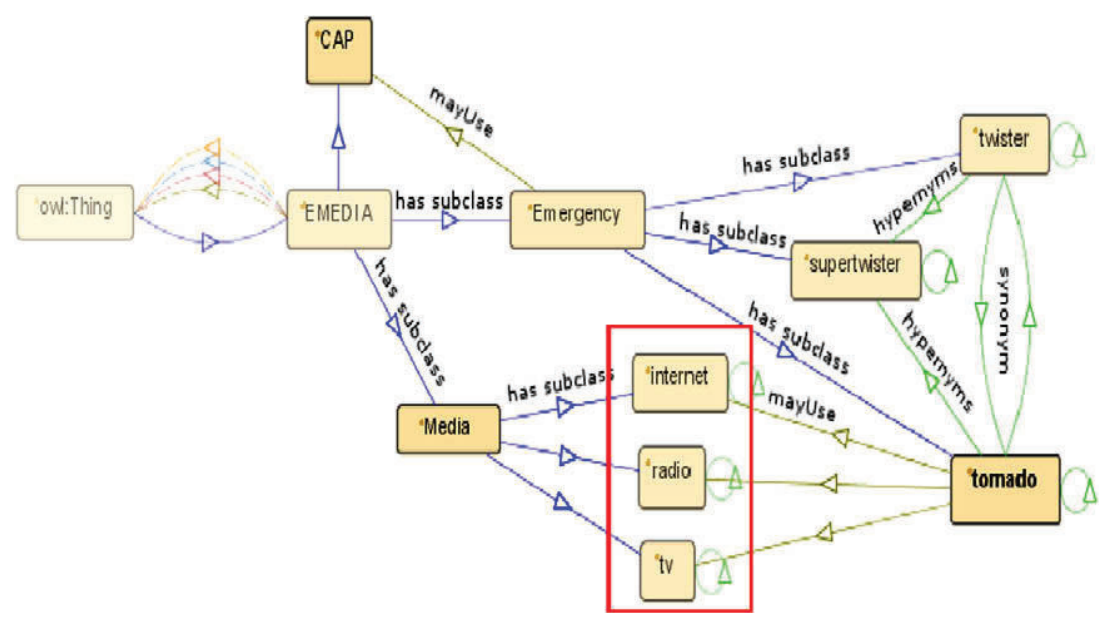

Fig. 4. Tornado and media that can be used to alert about.

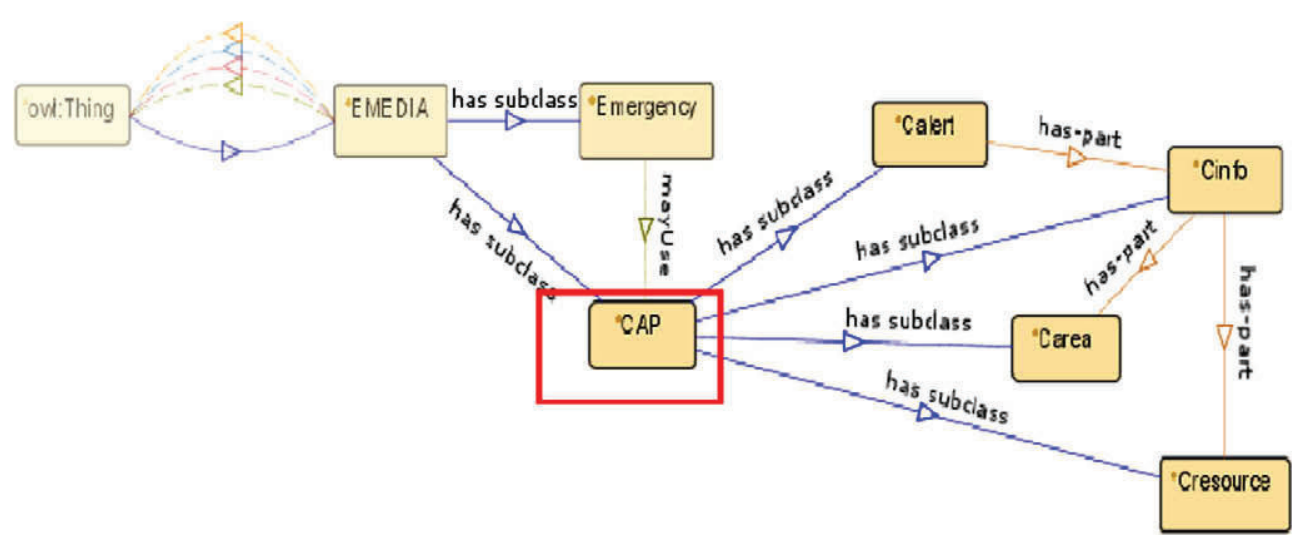

Fig. 5. Common alerting protocol description.

accessibility and supported devices, we considered the Web Content Accessibility Guidelines 1.0 with W3R Recommendation of 5 May 1999 and 24 articles from www.webaim.org (Web Accessibility in Mind).

We extracted all words from the corpus and applied a tagging procedure for the analysis of corpus texts. We analysed the input texts (the whole corpus in textual form) and tagged each word with its syntactic function (nouns, verbs, adjectives, etc.). We con sidered only nouns and verbs at this stage and reduced them to the root form (singular nouns and verbs at infinitive). This normalised corpus has been used to help in generating the lexons validation lists submitted to the evaluators (domain experts) and to act as a corpus to be quantitatively evaluated against lexons extracted from our ontology. In fact, we could compare lexons extracted from the corpus with lexons obtained from our ontology. Successively, we applied a second iteration where we filtered our ontology by using feedbacks of the evaluators and contrasted it against the cor pus to verify the accuracy of the contained information. 


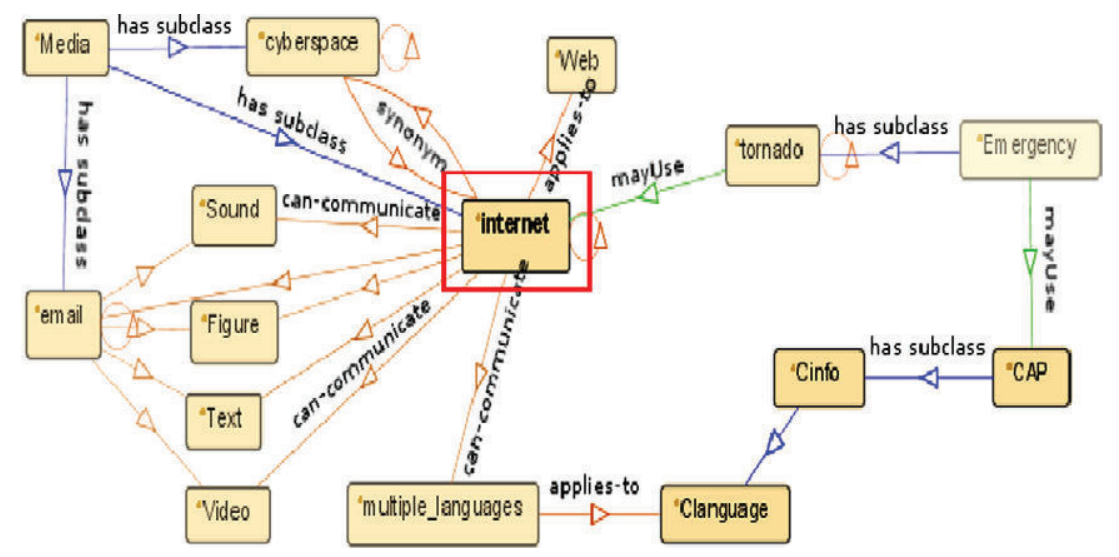

Fig. 6. Internet communication capabilities.

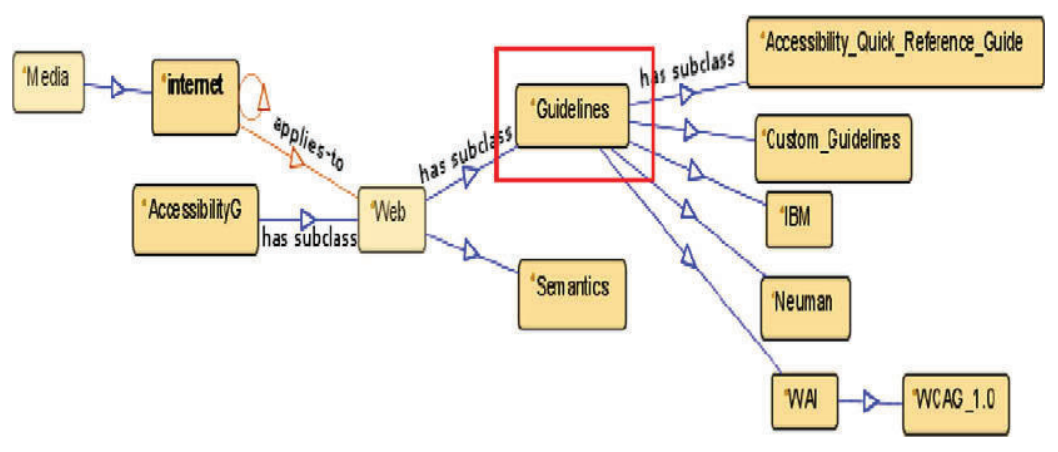

Fig. 7. Web accessibility guidelines.

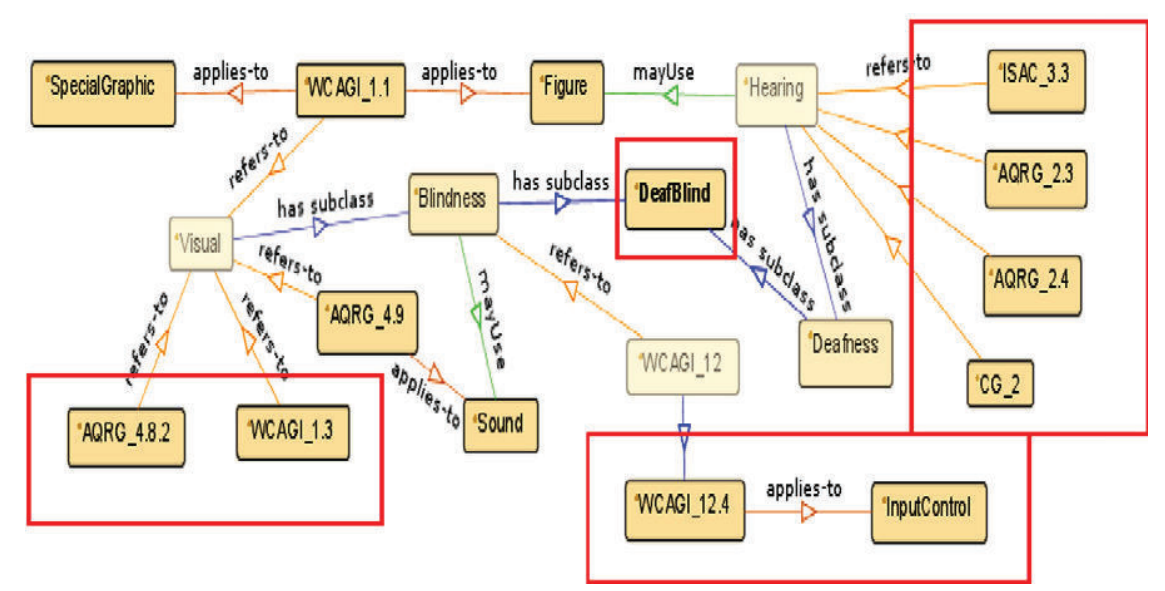

Fig. 8. Guidelines for deafblind.

\subsection{Qualitative method}

Domain experts were asked to evaluate the value and useful ness of the ontology extracted lexons in building a base knowledge for his/her domain. In fact, according to the HCOME Methodology (Kotis et al., 2006) knowledge workers should participate actively in the ontology engineering processes. We selected two evaluators: one is an expert of accessibility who worked several years for R\&D projects; she is particularly expert on Infometrics (information measurement) applied to web accessibility. The second evaluator is an expert professional working for Spanish Civil Protection and developing documents, policies and recommendations on the emergency domain.

Questions have been asked in form of a short evaluation ques tionnaire associated to the lexons list extracted from the ontology and for the respective domains. We reduced the number of pre sented lexons to hundreds of terms by matching the lexons con tained in the ontology with the ones also present in the 
normalised corpus. This is due to the fact that a human evaluator can be prompted with hundreds of terms and not with thousands (used in the quantitative evaluation).

It is important to notice that, to our knowledge, there are very few documents on both the fields, so about emergency and acces sibility together, while our ontology includes concepts from both domains.

Due to these reasons we generated two different lists, one with emergency related lexons evaluated by the emergency expert and another one with accessibility lexons evaluated by the accessibility expert.

The evaluator as a domain expert (professional in its field with years of experience), was prompted with three criteria to rate the lexons extracted from SEMA4A ontology.

- Coverage (Have all the lexons to be discovered actually been discovered?).

- Precision (Are the lexons making sense for the domain?).

- Accuracy (Are the lexons not too general but reflecting the important terms of the domain?).

Which specifically were translated in the following questions asked for each presented lexon:

- Is the lexon in the domain?

- What is the level of precision of the lexon?

- Does the lexon make sense for the specific domain?

We assigned different discrete values to the possible answers: $0 / 1$ for yes/no to the questions of type A; $0 / 1$ for yes/no to the ques tions of type $B$; and $1 / 2 / 3$ for specific/not too specific/general to the questions of type $C$.

The expert on accessibility evaluated, totally, 155 lexons ex tracted from our ontology in the accessibility domain.

Results were:

- Coverage $91 \%$ (Have all the lexons to be discovered actually been discovered?).

- Precision $84 \%$ (Are the lexons making sense for the domain?).

- Accuracy $79 \%$ (Are the lexons not too general but reflecting the important terms of the domain?). With Accuracy corresponding to "specific"; the expert also rated a $9 \%$ of "not too specific", and a $12 \%$ of "general" lexons.

The expert on emergency evaluated, totally, 265 lexons ex tracted from our ontology in the emergency domain.

Results were:

- Coverage 66\% (Have all the lexons to be discovered actually been discovered?).

- Precision 65\% (Are the lexons making sense for the domain?).

- Accuracy $45 \%$ (Are the lexons not too general but reflecting the important terms of the domain?). With Accuracy corresponding to "specific", while having a $1 \%$ of "not too specific", and a $54 \%$ of "general" lexons.

These results were mainly due to the fact that the emergency portion of our ontology was automatically built by extracting rele vant information from corpus of documents suggested by experts; while the accessibility part was built by integrating ontologies that were already verified and cleaned. Fig. 9 shows the average scores on experts' evaluations for accessibility and emergency compo nents of SEMA4A.

In the next session, we will show how we used the qualitative results to improve the ontology and thus increase accuracy and

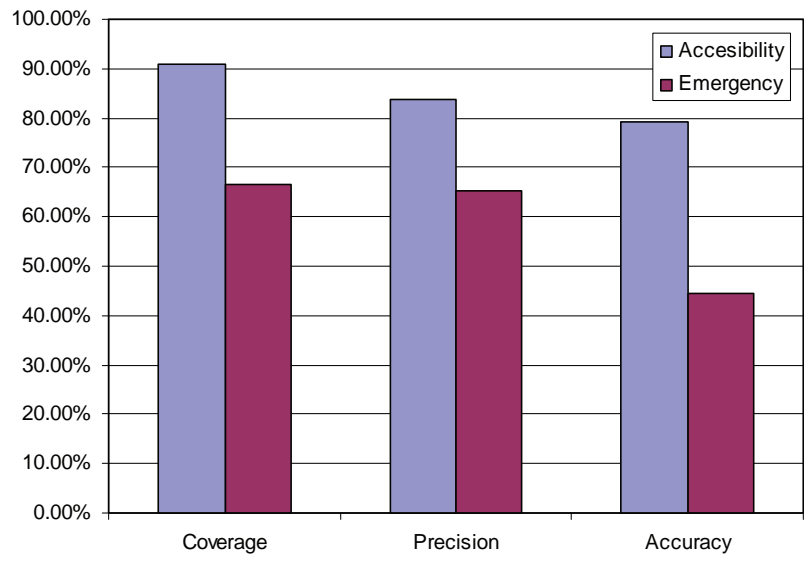

Fig. 9. Average scores on coverage, precision and accuracy as evaluated by experts on accessibility and emergency over lexons contained in the SEMA4A ontology.

coverage when measuring these characteristic with a quantitative method.

\subsection{Quantitative method}

In a previous paper (Malizia et al., 2008), we defined a quanti tative measure and semi automated evaluation procedure for mea suring coverage and accuracy of lexons over an entire corpus of documents. The procedure was inspired by Zipf's law (Zipf, 1949). The idea is that the frequency of occurrence of a word in a corpus of documents is inversely proportional to its frequency class; it means that words with higher frequency are less meaning ful for the corpus domain than words with less frequency (even if words with a low frequency can be too peculiar to be relevant for a domain within a corpus of documents).

Coverage has been measured by counting for each frequency class the number of lexon terms contained in the ontology that are identical with terms extracted from the corpus and comparing this number to the overall frequency class term count. Accuracy has been estimated on the basis of the coverage percentage for an interval of frequency classes. As the SEMA4A lexons consist of three words (two terms and one role), it is possible to investigate how much the produced lexons cover the corpus vocabulary, and more importantly how accurate they are. Regarding the accuracy, determining exactly which frequency classes contain the terms most characteristic for a domain still depends mainly on intuition and subjective opinions. It should also be point out that no stop word list has been defined because lexons have been produced extracting nouns and verbs.

In a previous preliminary work we presented the graphic repre sentation in Fig. 9 where the lowest $(<9)$ and highest $(>250)$ fre quency classes were omitted since did not contain relevant words (they contained too specific or too general terms for being relevant to display).

Fig. 10 shows that the coverage improved with the increasing rank of the frequency classes (until FC $=250$ to have a clear view of the graph). On average, the coverage ratio was $32.56 \%$. The accu racy (i.e. the coverage percentage for the selected interval) ratio for the 9250 intervals was $42.24 \%$. This last phenomenon was proba bly due to the fact that our corpus was made of documents about the different accessibility and emergency domains but including few existing documents on both domains and that had possibly re duced the specific terms but supported more general terms used in the technical domain senses.

In this work we introduced the qualitative evaluation of experts and so we used these evaluations to filter our ontology and clean it 


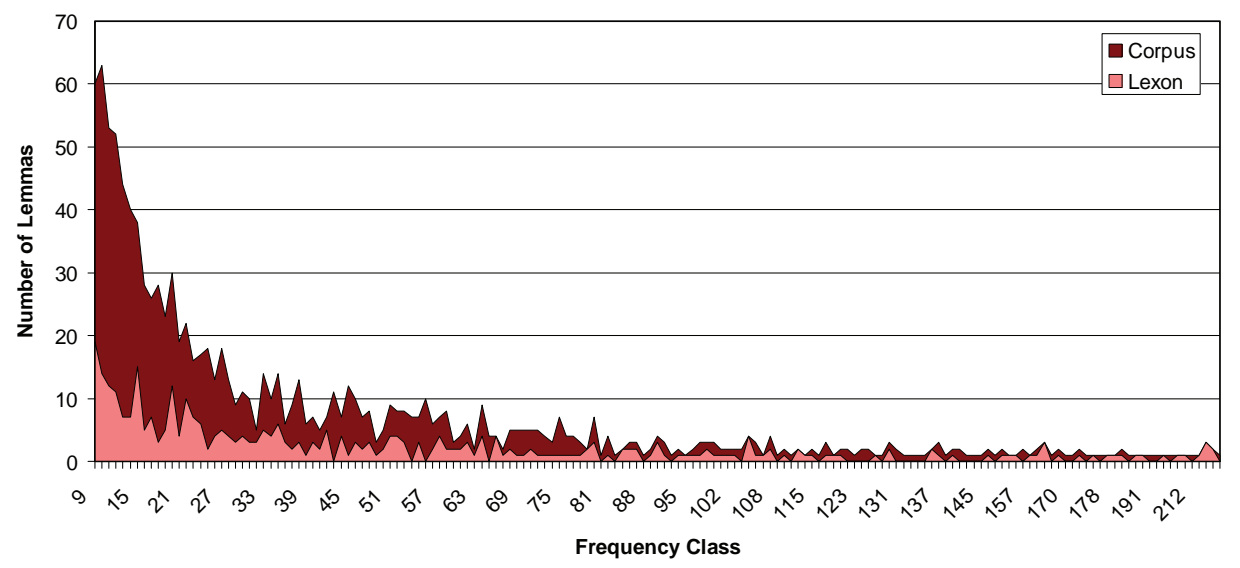

Fig. 10. Coverage and accuracy of frequency classes of the ontology over the corpus. Lemmas are the terms contained in each group of lexons (two for each lexon).

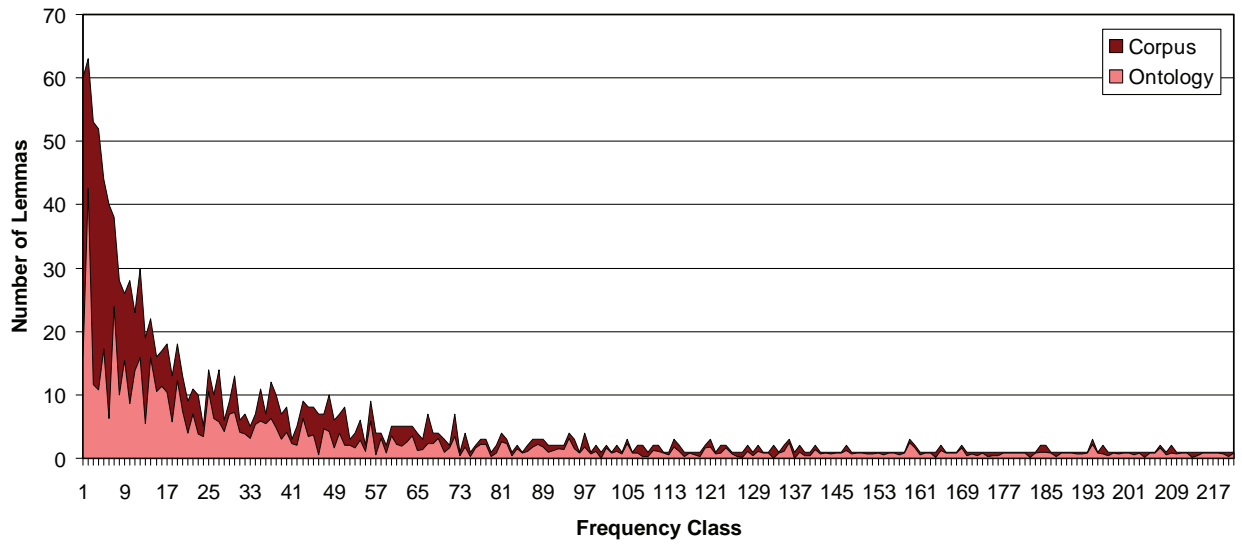

Fig. 11. Coverage and accuracy of frequency classes of the ontology over the corpus after filtering the terms by using the experts' evaluation. Lemmas are the terms contained in each group of lexons (two for each lexon).

from less specific or ambiguous terms that dropped down the accuracy and coverage of our previous approach (shown in Fig. 11).

We filtered out from our ontology terms that where judged too general or not specific for our domains by experts. Terms like "Link_location_attribute" or "Link_AccessKey" were judged too pe culiar by accessibility experts (even if included in guidelines im ported in our ontology); while terms like "novice" were too general for the accessibility domain. From the emergency point of view experts judged too general terms like "attention" or "re moval", while terms like "finite_quantity" or "unfortunate_person" were too peculiar for the emergency domain.

So, after this filtering phase, we ran again our evaluation proce dure onto the same corpus but with reduced set of concepts (many terms and thus lexons were filtered out following the qualitative evaluation). Thus, we had exactly the same ontology as before but filtered with the experts evaluation of too specific or too pecu liar terms.

Fig. 11 shows that the coverage improved with the filtered rank of the frequency classes (until FC $=250$ to have a clear view of the graph). On average, the coverage ratio was $70.04 \%$. The accuracy (i.e. the coverage percentage for the selected interval) ratio for the 1100 intervals was $74.42 \%$.

The frequency classes are lower than in the previous experi ment since this time, after filtering, we have words that were much more relevant for the domain and so for the Zipf law they were all compressed within lower frequency classes; while, again for the same law, the number of lemmas was higher.

\section{Conclusion}

When critical events can occur people has to be informed with complete and understandable information to reduce the damages or to inform about what measures can be taken for peoples' safety. In fact, as stated by Spanish Civil Protection: "Everywhere, at every time an unexpected critical event can occur damaging people or things" (DGPCE, 2007).

We showed that emergency information systems generally do not include information and knowledge about different kind of users to be notified about an emergency; there is a lack in taking into account differences in accessing the information sources (e.g. Internet or radio) or available resources depending on the cog nitive and physical abilities of people. Providing accessible infor mation within emergency notification systems is crucial, since, it can reduce the number of victims and strongly help users in receiv ing emergency and critical news.

To solve this problem of communicating emergencies and crit ical information to different categories of users (impaired, aged, ...) within different kind of emergencies using different technolo gies, we have developed a knowledge base codified as an ontology. We have also explored accessibility in general and investigated on broad guidelines and users' experts guidelines coded as ontologies. We selected two ontologies: WAfA, and AccessOnto considering them as the most relevant for codifying accessibility concepts in emergency scenarios. We, then, developed another portion of our ontology called EMEDIA with a semi automated technique includ 
ing information on emergencies, and how they could affect technologies.

Starting from the three portions we have developed a new ontology called SEMA4A including concepts and relationships con sidering different users profiles and abilities in conjunction with different communication medias (EMEDIA) and accessibility guidelines.

The novelty of our approach consists of providing accessible notifications within ERIS. Furthermore we also included interoper ability features both at syntactic level (supporting OWL and stan dard languages) and at semantics level by mapping and linking concepts coming from different fields (accessibility, emergency, multimedia) into the same ontology.

We used a qualitative evaluation to test whether our ontology included the most relevant concepts in the domain of ERIS and accessibility. We obtained results from experts in both domains (emergency and accessibility) verifying that we have enough cov erage on both the fields; thus, our ontology could be employed for developing systems that could infer information to automati cally adapt emergency notifications depending on the recipients' characteristics.

Furthermore we employed a quantitative evaluation technique measuring the coverage and accuracy of our ontology over a corpus of documents (on accessibility, devices and emergencies) sug gested by domain experts. We may notice that even if we had around $70 \%$ of coverage and $74 \%$ of accuracy, the corpus was mainly separated into two topics and only few documents shared the topics of accessibility and emergencies (this is exactly the lack of information we are trying to fill with our work). Nevertheless with the help of experts' evaluation we improved the coverage and accuracy of our ontology with respect to our previous work.

Future works include the possibility of integrating the ontology within an emergency system to test whether effective notification can be generated by an event driven process refined by the knowl edge base contained in the ontology to inform users according to their devices and abilities. In fact, the knowledge codified in SE MA4A can be used at different levels:

- At static level as a set of facts and reusable content made by metadata (owl files) and possibly generating shared knowledge in for of HTML or XML files that could be used by different sys tems to interoperate.

- At dynamic level as a knowledge base for developing web ser vices (or semantic web services) able to query the knowledge base and derive actions to perform depending on the informa tion codified in SEMA4A.

Moreover we will automate such process by developing a fine grained level in the ontology to exactly match users' abilities with accessibility guidelines and new interactive media features, such as: touch sensitive, tactile, force feedback (force/resistance), tex ture, heat, vibration, etc.

We are also considering the evolution of the ontology when used by knowledge workers in emergency domain. The evolution of the ontology can be related to the evolution of its schema, struc ture, and the introduction of updated knowledge (Noy and Klein, 2004).

Finally we aim to validate our ontology within a wider audience of domain experts testing it within international organizations. In fact, we will use our ontology integrating it within a system (SI GAME $^{15}$ ) managing real emergencies as a test bed among a commu nity of users interoperating for solving emergency scenarios.

\section{Acknowledgments}

This work has been partly funded by UIA4SIGE (Ministerio de Educación y Ciencia TSI2007 60388) and MDDSIGE (CAM UC3M CCG06 UC3M/TIC 0787) projects. Authors also want to acknowl edge F. Astorga Paliza for his comments and previous collaboration.

\section{Appendix A. SEMA4A classes}

In this appendix we give a brief description of the main classes and concepts included in the ontology to give an overall idea of the specific knowledge codified in SEMA4A. The main classes, as pre sented in Fig. 1, are: EMEDIA (including concepts and relations on emergency and communication devices and technologies), AccessOnto (including concepts on disabilities and accessibility guidelines), and WAFa (including structural definitions for format ting content on the web, to which usually guidelines are applied).

\section{A.1. EMEDIA}

EMEDIA (Emergency and MEDIA technologies) is the portion of the SEMA4A ontology that provides concepts and relations about emergency and media technologies. We developed it trough a semiautomatic procedure with two phases: the first phase was performed to extract new concepts and relations from WordNet (concerning emergency and media technologies); the second to integrate new information within the existing ontology (adding relations with the others portions). We applied this technique to develop and expand part of our ontology related to the emergen cies and how they can affect technologies accessible to the users.

The considered corpus is composed by articles about emer gency, accessibility and devices as suggested by the domains ex perts. In particular, for the emergency topic there are about sixty seven article from the proceedings of the conference ISCRAM2007 (Intelligent Systems for crisis management), plus a manual developed by the North Central Texas regional government (Know what to do. Think. Prepare. Act. ${ }^{16}$ ) and papers on community emergency man agement (for example: Schafer et al., 2007). For accessibility and supported devices, we considered the Web Content Accessibility Guidelines 1.0 with W3R Recommendation of 5 May 1999 and 24 articles from http://www.webaim.org (Web Accessibility in Mind). The total number of analysed words is about 300,000 for 500 pages.

\section{A.2. WAfA}

Web Authoring for Accessibility (WAfA) is an existing ontology also known as Travel Ontology because it is based on the analogy of web navigation with tourists' trips. This ontology represents con cepts and relations necessary to automatically model the structural organization and navigation of web pages (Yesilada, 2005) to users' profiles.

This ontology has been evaluated with real users, contains information on how to model content for being accessible, and it is codified using OWL; we extended our ontology including WAfA concepts, defining a class called WAfA that contains concepts and relations needed to model organization, structure and navigation of sites.

\section{A.3. AccessOnto}

AccessOnto in an ontology in form of an accessibility require ments repository from which it is possible to extract requirements

\footnotetext{
${ }^{16}$ http://www.knowwhat2do.com/en/pdf/KnoWhat2Do_Guide.pdf.
} 
using an accessibility knowledge base (AKB) built on user's charac teristics (Masuwa Morgan and Burrell, 2004). It includes guide lines from Web Accessibility Initiative, Sun Micro Systems, IBM, Microsoft, and Apple guidelines.

In our ontology we created a class called AccessOnto that con tains information related to Web accessibility guidelines, users' profiles and actions that users can perform. We created this class translating information from XML (AccessOnto is codified in $\mathrm{XML}$ ) to OWL; after this phase, we established relations that linked concepts contained in WAfA and in AccessOnto sections, as we will show in the use case section.

Summarizing, SEMA4A counts on three basic classes: WAfA, AccessOnto and EMEDIA; including information related to con cepts and relations needed to model organization, structure and navigation of information contents; accessibility guidelines, user's profiles and actions that users can perform; as well as information related to emergencies, notifications and devices. These main clas ses are linked with relations existing within their subclasses. The following section we provide a use case that depicts the more com mon relations that exist in our ontology.

\section{References}

A-Prompt (2006). University of Toronto, A-Prompt. <http:// aprompt.snow.utoronto.ca/> Retrieved 14.03.06.

Addams-Moring, R., Kekkonen, M., \& Zhao, S. (2005). A simple taxonomy for mobile emergency. In B. Van de Walle \& B. Carlé (Eds.), Proceedings of the 2nd international ISCRAM conference, Brussels, Belgium, April 2005 (pp. 309-316).

Berners-Lee, T. (2007). Disaster management ontologies? [Online] <http:// lists.w3.org/Archives/Public/semantic-web/2007Apr/0094.html> Retrieved June, 2007.

Bobby (2006). Watchfire Corporation, Bobby. <http://bobby.watchfire.com/> Retrieved 14.03.06

Burzagli, L., Emiliani, P., \& Gabbanini, F. (2009). Design for all in action: An example of analysis and implementation. Expert Systems with Applications, 36(2), 985-994. Part 1, March.

Cristea, A. I. (2004). What can the Semantic web do for adaptive educational hypermedia? International peer-reviewed on-line journal. Educational Technology and Society, 7(4), 40-58. (Special Issue on Ontologies and the Semantic Web for E-Learning. IEEE, LTSC).

Dix, A., Finley, J., Abowd, G., \& Beale, R. (2003). Human-computer interaction (3rd ed.). Prentice-Hall, Inc..

DGPCE - Dirección General de Protección Civil y Emergencias, Spain (2007). Conocimientos generales sobre protección civil. <http:// www.proteccioncivil.org/centrodoc/publicaciones/conogralpc.pdf> Retrieved April 2007.

Geller, J., Perl, Y., \& Lee, J. (2004). Editorial: Ontology challenges: A thumbnail historical perspective. Knowledge and Information Systems, 6(4), 375-379.

Gabrielli, S., Mirabella, V., Kimani, S., \& Catarci, T. (2004). Steering the development of accessible e-learning content. In Proceedings of the 3rd ECEL conference, Paris (F) (pp. 517-526).

Gabrielli, S., Mirabella, M., Teso, M., \& Catarci, T. (2005). The design of an authoring interface to make e-learning content accessible. Interact, 8, 1100-1103.
Guarino, N. (1997). Understanding, building, and using ontologies: A commentary to using explicit ontologies in KBS development", by van Heijst, Schreiber, and Wieling. International Journal of Human and Computer Studies, 46, 293-310.

ICTSB (2000). The ICT Standards Board Project Team. Design for all, final report. $<$ http://www.ictsb.org/>.

Kotis, K., \& Vouros, A. (2006). Human-centered ontology engineering: The HCOME methodology. Knowledge and Information Systems, 10, 109-131.

LiFT (2006). UsableNet, LiFT. <http://www.usablenet.com/> Retrieved 14.03.06.

Lozano-Tello, A., Macías, M., Sánchez, F., \& Sosa, E. (2003). Uso de Ontologías en Páginas Web para Mejorar su Accesibilidad a Invidentes. VIII Jornadas de Ingeniería del Software y Base de Datos (JISBD’03), Alicante, Spain (pp. 625634)

Lozano-Tello, A., Macías, M., Prieto, A., Sánchez, F., \& Sosa, E. (2004). Contenidos Web Accesibles a Invidentes Meditante Ontologías. II Congreso Virtual “Derecho y Discapacidad en el Nuevo Milenio Cáceres, 17-18 December 2004.

Malizia, A., Astorga-Paliza, F., Onorati, T., Díaz, P., \& Aedo, I. (2008). Emergency alerts for all: an ontology based approach to improve accessibility in emergency alerting systems. In Proceeding of the 5th International Conference on Information Systems for crisis response and management (ISCRAM 2008) (Vol. 1, pp. 197-207).

Masuwa-Morgan, K. R., \& Burrell, P. (2004). Justification of the need for an ontology for accessibility requirements (theoretic framework). Interacting with Computers, 16(3), 523-555.

Miller, G. A., Beckwith, R., Felbaum, C., Gross, D., \& Miller, K. (1990). Introduction to WordNet: An on-line lexical database. International Journal of Lexicography, 3(4), 235-244.

Neches, R., Fikes, R., Finin, T., Gruber, T., Patil, R., Senatir, T., et al. (1991). Enabling technology for knowledge sharing. AI Magazine, 12(3), 36-56.

Noy, N. F., \& Klein, M. C. A. (2004). Ontology evolution: Not the same as schema evolution. Knowledge and Information Systems, 6(4), 428-440.

Pattuelli, M. C. (2008). Teachers' perspective and contextual dimensions to guide the design of N.C. history learning objects and ontology. Information Processing and Management, 44(2), 635-646.

Pinto, H. S., \& Martins, J. P. (2002). Ontologies: How can they be built? Knowledge and Information Systems, 6(4), 441-464.

QUERCUS (2007). Software Engineering Group. <http://quercusseg.unex.es>.

Schafer, W. A., Ganoe, C. H., \& Carroll, J. M. (2007). Supporting community emergency management planning through a geocollaboration software architecture. Journal of Computer Supported Cooperative Work (CSCW), 16(4-5), 501-537.

Sowa, J. F. (1984). Conceptual structures: Information processing in mind and machine. Addison-Wesley.

Spyns, P., Meersman, R., \& Jarrar, M. (2002). Data modelling versus ontology engineering. SIGMOD Record, 31(4), 12-17 (Special Issue).

Van de Walle, B., \& Turoff, M. (2007). Emergency response information systems: Emerging trends and technologies. Communications of ACM, 50(3), 29-31.

WAI (2006). Web Accessibility Initiative. <http://www.w3.org/WAI/>

Yang, S. J. H., \& Shao, N. W. Y. (2007). Enhancing pervasive Web accessibility with rule-based adaptation strategy. Expert Systems with Applications, 32(4), 1154-1167.

Yesilada, Y., Harper, S., Goble, C., \& Stevens, R. (2004). Screen readers cannot see (ontology based semantic annotation for visually impaired web travellers). In Nora Koch, Piero Fraternali, \& Martin Wirsing, (Eds.), Web engineering - 4th international conference, ICWE 2004 Proceedings, LNCS 3140 (pp. 445-458). Springer, 2004.

Yesilada, Y. (2005). Annotation and transformation of Web pages to improve mobility for visually impaired users. PhD thesis. School of Computer Science at the University of Manchester, UK.

Zipf, G. K. (1949). Human behaviour and the principle of least-effort. Cambridge, MA Addison-Wesley. 